\title{
Groin pain - uncommon presentation of common entity with concomitant occurrence of three mimickers funiculitis, inguinal hernia and spermatic cord lipoma
}

\author{
Authors \\ Amit Kumar Paliwal MD (Radiodiagnosis) ${ }^{1}$, Saurov Ghose MS (Gen Surgery) ${ }^{2}$, \\ Brajesh Kumar MS,DNB (Gen Surgery) ${ }^{3}$, \\ Somali Pattanayak MD, DNB (Radiodiagnosis) ${ }^{4}$ \\ Military Hospital Dehradun, Garhi Cantt, Dehradun, Uttrakhand, India, Pin: 248003
}

\begin{abstract}
Groin pain is a common condition in young males with varied differentials. Inguinal hernia, funiculitis and spermatic cord lipoma are relatively common with different management strategies. Thus reaching a definitive diagnosis on clinico-radiological profile is essential. We present a case of groin pain with simultaneous presence of three mimickers.
\end{abstract}

Keyword: Groin pain, Funiculitis, inguinal hernia, spermatic cord lipoma.

\section{Introduction}

Funiculitis or vasitis mimic indirect inguinal hernia leading to inadvertent surgical intervention. Spermatic cord lipmoa is also a close differential for these two conditions. We present a case of young male presented with left lower abdominal and groin pain where imaging demonstrated funiculitis and indirect hernia and during the repair of hernia a spermatic cord lipoma was also detected.

\section{Case Report}

A 34 year male reported to hospital with 7-10 days history of left lower abdominal and groin pain, clinical examination revealed tenderness in left iliac fossa with no obvious mass or lump. Patient was advised ultrasound scan abdomen which revealed echogenic omental fat in left iliac fossa (Figure 1) with probe tenderness in this region, possibility of diverticulitis and Epiploic appendagitis were given and patient was advised CECT abdomen. CECT revealed significant omental fat stranding in left lower abdomen with mild circumferential bowel wall thickening of Sigmoid colon and Descending colon, omental fat stranding was disproportionate for the bowel wall abnormality. Also there was widening of left deep inguinal ring with herniation of omentum (revealing fat standing) in to the inguinal canal, the left spermatic cord was also enlarged (Figure 2). Inguinoscrotal ultrasound was done for further correlation which revealed thickened hyperechoic spermatic cord with widening of deep inguinal ring and herniation of omentum increasing on performing valaslva manoeuvre or coughing, the testis and epididymis were normal. Thus an impression of left sided funicultis and indirect inguinal hernia were given with possible omental infarction along with reactive 
bowel wall thickening. Patient was taken up for surgery for repair of hernia where the findings of indirect hernia and funiculitis were confirmed and additionally a cord lipoma was also discovered (Figure 3). The hernia was repaired and lipoma was excised. Follow up NCCT scan after 45 days revealed significant resolution of fat stranding, resolution of bowel wall thickening, mild spermatic cord thickening and no recurrence of hernia (Figure 4).

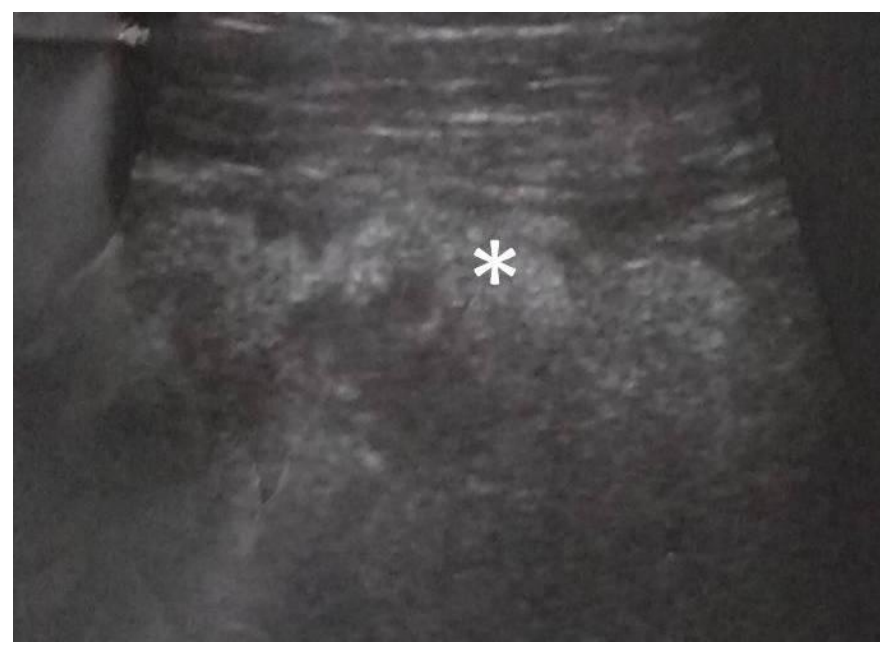

Fig. 1 USG abdomen hyperechoic omental fat (white star).

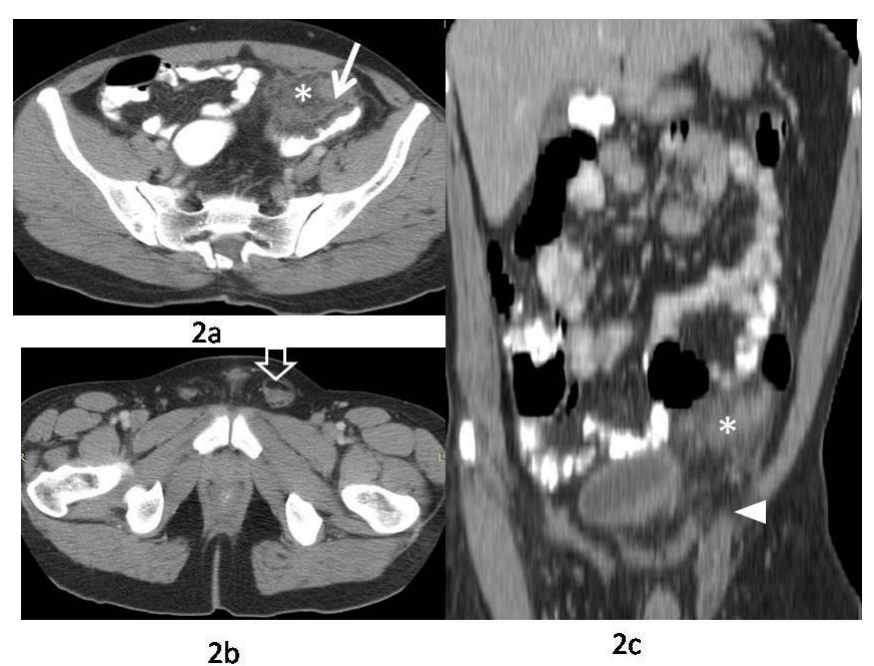

Fig.2 CECT abdomen

2a: Axial CECT image: omental fat stranding (white star) with mild circumferential bowel wall thickening of Sigmoid colon (white arrow).

2b: Axial CECT image: widening of left inguinal canal (white arrow head) containing thick left spermatic cord (posteriorly) and fat attenuating contents (anteriorly). 2c: Coronal CECT image: inguinal hernia (white arrow head) with thick left spermatic cord (curved white arrow) and omental stranding (white arrow).

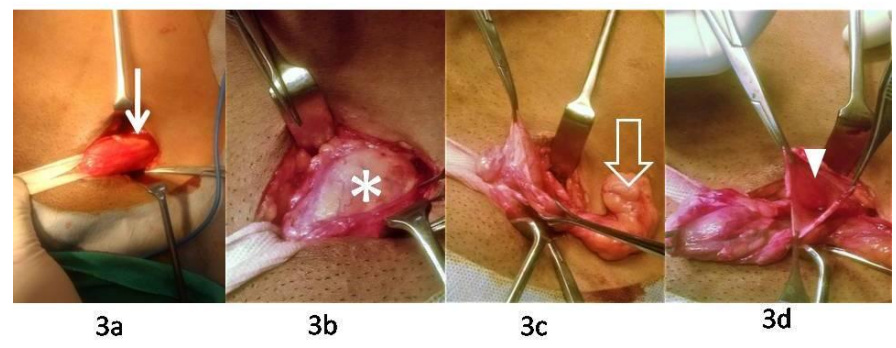

Fig 3 intra-op images

3a: Thick left spermatic cord (white arrow).

3b: spermatic cord lipoma (white star).

3c: Spermatic cord lipoma post resection

(open arrow)

3d: Hernia sac (white arrow head)

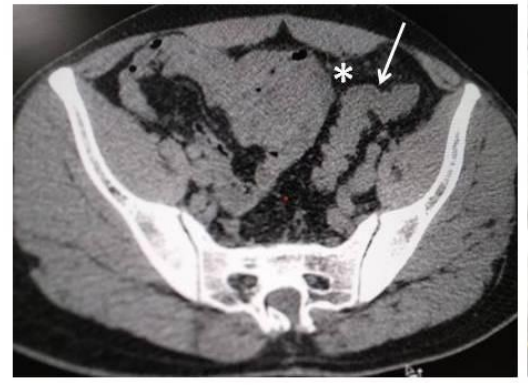

4a

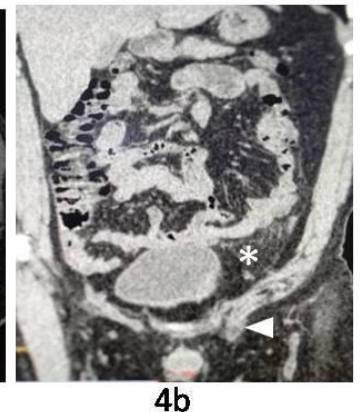

$4 b$
Fig 4 NCCT abdomen after 45 days 4a: Axial NCCT image: significant resolution of omental fat stranding (white star) and Sigmoid colon wall thickening (white arrow).

4b: Coronal NCCT image: Mildly thickened left spermatic cord (curved white arrow) and minimal omental fat stranding (white arrow). Opposed left deep inguinal ring (white arrow head).

\section{Discussion}

Funiculitis or vasitis is the inflammation of spermatic cord which closely mimics an incarcerated inguinal hernia ${ }^{[1]}$. Funiculitis is relatively rare as compared to inguinal hernia and inadvertent surgeries has been performed in past in cases of funiculitis considering it to be an incarcerated inguinal hernia. Clinically in both conditions patient present with groin pain and mass, imaging including USG/CT or MRI may help in differentiating these two conditions where thick 
edematous spermatic cord is demonstrated in funiculitis while in incarcerated hernia widening of inguinal canal and hernia contents are demonstrated ${ }^{[2]}$. Imaging also helps in excluding other possible differentials like epididymitis and testicular torsion [2]. In our case patient presented with left sided lower abdominal and gorin pain without obvious mass or lump which is relatively unusal in funiculitis and hernia. Also the initial USG scan was only done for abdomen which showed hyperechoic omentum and further CECT of abdomen showed presence of funiculitis and inguinal hernia with omental stranding and subjacent bowel wall thickening which is again unusal in both conditions, since the bowel thickening was disproportionate to omental stranding it was thought to be reactive secondary to omental infarction ${ }^{[3]}$ which might have occurred due strangulation of omentum due to hernia or inflammatory changes secondary to funiculitis. Treatment of choice in funiculitis is conservative medical management while in inguinal hernia surgical repair has to be done thus it is essential to differentiate these two conditions ${ }^{[2]}$. As per our knowledge simultaneous presence of these two mimickers with presurgical imaging diagnosis has not been described. Our patient underwent mesh repair for indirect hernia where a cord lipoma was also observed apart from hernia and funiculitis, thus making our case very unique where three mimickers were present simultaneously. Reduction of fat stranding and resolution of bowel wall thickening post hernia repair and conservative management of funiculitis confirmed that these were secondary to hernia and/or funiculitis.

\section{Conclusion}

Groin pain is a common condition in young males with varied differentials. Inguinal hernia, funiculitis and spermatic cord lipoma are relatively common with different management strategies. Thus reaching a definitive diagnosis on clinico-radiological profile is essential to avoid inadvertent surgical intervention. Very rarely these mimickers may occur simultaneously as described in this case report making clinical and imaging diagnosis difficult.

\section{References}

1. K Walid et al. Vasitis: An Uncommon Diagnosis Mimicking Incarcerated Inguinal Hernia. Urology, 2016 Jul; 93: e1.doi.org/ 10.1016/ j.urology. 2016.03.011

2. Eddy K, Connell D, Goodacre B, Eddy R. Imaging findings prevent unnecessary surgery in vasitis: an under-reported condition mimicking inguinal hernia. Clin Radiol. 2011 May;66(5):475-7. doi: 10.1016/j.crad.2010.12.006.

3. Tonerini M, Calcagni F, Lorenzi S, Scalise P, Grigolini A, Bemi P. Omental infarction and its mimics: imaging features of acute abdominal conditions presenting with fat stranding greater than the degree of bowel wall thickening. Emerg Radiol. 2015 Aug;22(4):431-6. doi: 10.1007/s10140-0151302-0. Epub 2015 Mar 1. 